\title{
Determining the optimum way to maintain quality of life for very elderly patients with advanced bladder cancer and poor performance status: A case report
}

\author{
KENJIRO SUZUKI $^{1,2}$, FUMINARI HANASHIMA ${ }^{1}$, SUGURU SHIROTAKE $^{1}$, KIICHIRO KODAIRA ${ }^{1}$, \\ KOSHIRO NISHIMOTO ${ }^{1}$, TAKAO TAKAHASHI ${ }^{3}$, HIDEKI ONISHI ${ }^{4}$ and MASAFUMI OYAMA ${ }^{1}$ \\ ${ }^{1}$ Department of Uro-Oncology, Saitama Medical University International Medical Center, Saitama 350-1298; \\ ${ }^{2}$ Department of Urology, Suzuki Clinic, Chiba 289-2102; ${ }^{3}$ Departments of Palliative Medicine and \\ ${ }^{4}$ Psycho-Oncology, Saitama Medical University International Medical Center, Saitama 350-1298, Japan
}

Received October 25, 2016; Accepted April 4, 2017

DOI: $10.3892 / \mathrm{mco} .2017 .1236$

\begin{abstract}
Locally advanced bladder cancer causes unpleasant symptoms such as irritative voiding symptoms, lower abdominal pain, gross hematuria and urinary retention, and lowers the quality of life. Treatment decisions in elderly patients may be difficult, as elderly patients are physically and psychologically different from younger patients. An 89-year-old male was referred to hospital for the treatment of an invasive bladder tumor with right hydronephrosis from tumor obstruction. The patient was elderly and did not have a good performance status; therefore curative radical cystectomy or chemotherapy was not indicated. Left retroperitoneoscopic ureterocutaneostomy was performed to alleviate gross hematuria and voiding difficulty. Intensity-modulated radiotherapy was administered 9 days after the surgery to control bleeding in the bladder tumor. After completing 8 days of radiotherapy, the patient was discharged from hospital. The patient exhibited no signs of either postrenal failure or gross hematuria for 7 months prior to mortality. Retroperitoneoscopic ureterocutaneostomy for very elderly patients with advanced bladder cancer with a poor performance status may be an important procedure for alleviating symptoms and improving quality of life.
\end{abstract}

\section{Introduction}

Age is now widely accepted as the greatest single risk factor for developing bladder cancer. Bladder cancer is primarily considered a disease of the elderly due to the close link between age and incidence of bladder cancer (1). Treatment

Correspondence to: Dr Kenjiro Suzuki, Department of Uro-Oncology, Saitama Medical University International Medical Center, 1397-1 Yamane, Hidaka, Saitama 350-1298, Japan

E-mail: bashi510@yahoo.co.jp

Key words: retroperitoneoscopic ureterocutaneostomy, palliative treatment, invasive bladder cancer, gross hematuria, elderly patient decisions in elderly patients may sometimes be challenging, as elderly patients are physiologically, psychologically and socially different from younger patients.

The main symptoms of invasive bladder cancer are irritative voiding symptoms, lower abdominal pain, gross hematuria, resulting in anemia and urinary retention. Locally advanced tumors may be accompanied by several debilitating symptoms, including bleeding, pain, dysuria and urinary obstruction $(2,3)$. Although radical cystectomy is the standard treatment for localized muscle-invasive bladder cancer, chemotherapy is indicated for patients with advanced or metastatic bladder cancer, who are not candidates for surgery. However, the chemotherapy regimens are toxic and difficult to administer to elderly patients with a World Health Organization performance status 3 or 4 , with or without additional negative prognostic factors. The most appropriate approach for such patients is best supportive care; they may also be candidates for palliative treatment.

Occasionally, urinary diversion should be used, depending on the clinical condition of the patient. Ureterocutaneostomy is a urinary diverting procedure suitable for obstructive uropathy in patients with advanced pelvic malignancy, and is a less invasive approach for such poor-risk patients requiring palliation $(4,5)$. However, there have been fewer studies on ureterocutaneostomy for elderly patients. The present study reports a case of a very elderly patient with locally advanced bladder cancer that was refractory to medical management of severe irritative voiding symptoms, and was found be highly responsive to palliative urinary diversion by retroperitoneoscopic ureterocutaneostomy.

\section{Case report}

An 89-year-old male visited a local hospital with the chief complaint of gross hematuria and voiding difficulty. The patient had no past medical history and was not taking any anticoagulants. Computed tomography of the abdomen and pelvis showed a tumor on the right wall of the bladder and a right hydronephrotic atrophic kidney caused by tumor obstruction. The serum creatinine level was elevated to $1.44 \mathrm{mg} / \mathrm{dl}$ 


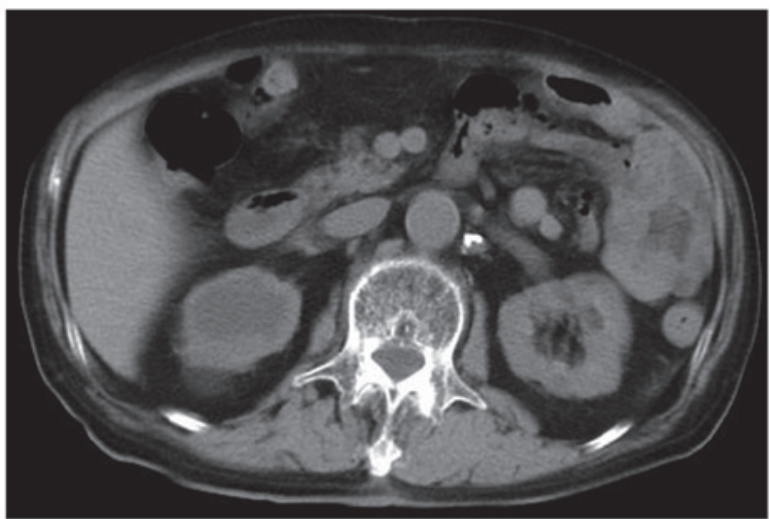

Figure 1. Computed tomography scan showing right hydronephrosis and a thin renal cortex resulting in a right atrophic kidney.
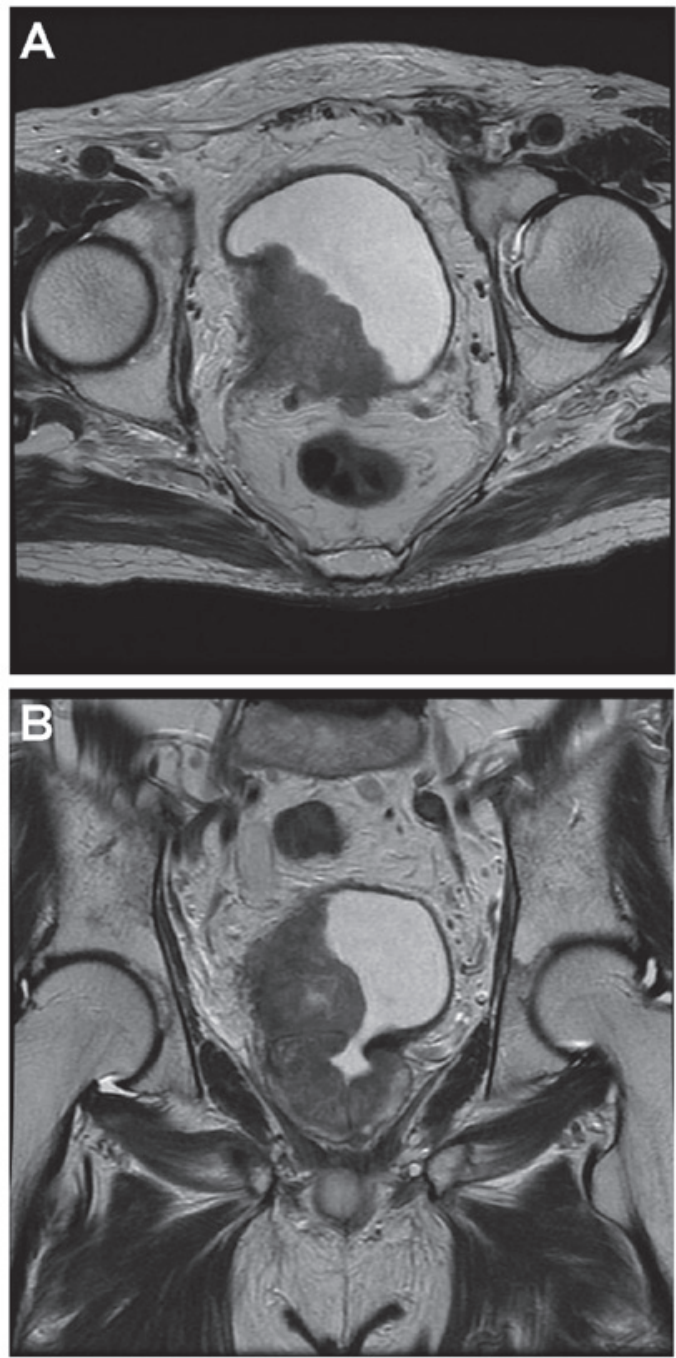

Figure 2. Magnetic resonance image showing a tumor on the right wall of the bladder, obstructing the right orifice and invading the perivesical tissue macroscopically. (A) Axial slice and (B) coronal slice.

(normal range, 0.43-1.08 $\mathrm{mg} / \mathrm{dl}$ ) (Fig. 1). Magnetic resonance imaging showed that the bladder tumor was invading the perivesical tissue macroscopically (Fig. 2). The patient was referred to Saitama Medical University International Medical Center (Saitama, Japan) for treatment.
A

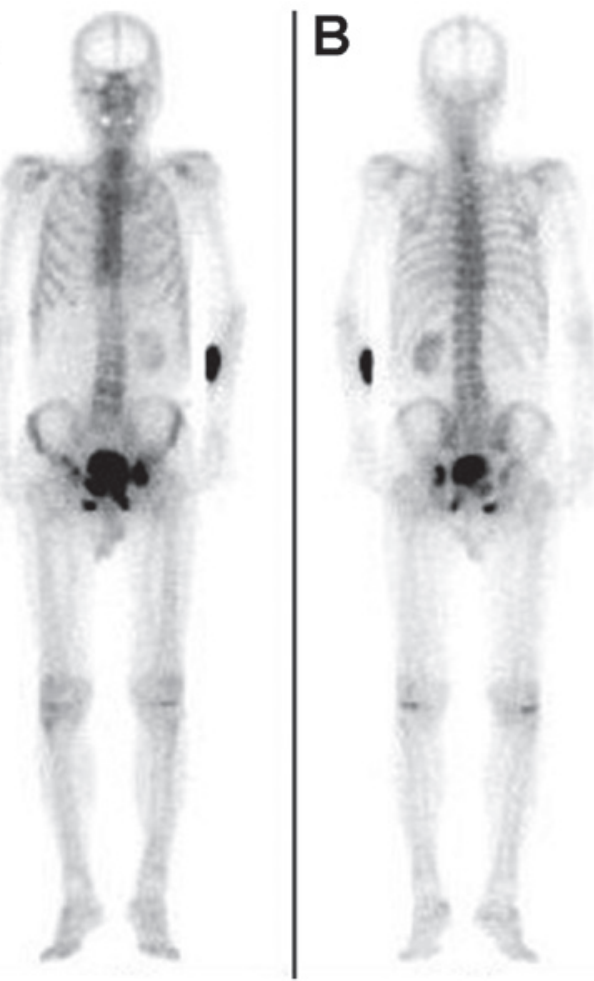

Figure 3. Bone scintigraphy revealing multiple bone metastases in the ilium, pubis and ischium. (A) Anterior view and (B) posterior view.

Bone scintigraphy revealed multiple bone metastases in the ilium, pubis and ischium (Fig. 3). Curative radical cystectomy was not indicated in this case, as distant metastases were present. The patient was elderly and did not have a good performance status, therefore neither cystectomy for palliation or chemotherapy was indicated. Palliative left retroperitoneoscopic ureterocutaneostomy was performed to improve renal function and relieve tumor bleeding without chemotherapy.

The surgery lasted 144 min and blood loss was kept to a minimum; a drain was left in place for $72 \mathrm{~h}$. A 7 FR ureteral stent was placed to prevent an anastomotic stricture. The patient started to walk and eat on the first postoperative day without postoperative delirium. The serum creatinine level was $1.37 \mathrm{mg} / \mathrm{dl}$ on the 7 th postoperative day; it remained similar to the preoperative level. The patient required a prescription for $12.5 \mu \mathrm{g} / \mathrm{h}$ fentanyl patches for severe pain in the right buttock and thigh regions. Intensity-modulated radiotherapy of $30 \mathrm{~Gy}$ in 10 fractions was administered 9 days after the surgery, to control tumor bleeding. After completing 8 days of radiotherapy, the patient was discharged from hospital.

Following discharge, the patient was able to spend the remainder of his life with no signs of either postrenal failure or gross hematuria. The pain control was adequate with fentanyl patches. The patient died due to progressive disease 7 months after the operation.

\section{Discussion}

The present case illustrated that urinary diversion by retroperitoneoscopic ureterocutaneostomy as a palliative treatment for a very elderly patient with invasive bladder cancer patient poor performance status was able to alleviate gross hematuria 
and voiding difficulty and to avoid possible complications due to progressive disease such as uncontrolled hematuria, urinary retention and postrenal failure due to obstructive uropathy. As a result, the patient maintained quality of life until they died.

Severe localized problems may occur in patients with invasive, inoperable bladder cancer and in those who have not undergone cystectomy due to metastatic disease. These problems include pain, bleeding, voiding problems and obstruction of the upper urinary tract. Patients' quality of life is markedly decreased due to such problems $(6,7)$. Salvage cystectomy is indicated for palliation of such symptoms; however, elderly patients and certain high-risk patients are not suitable for surgery. However, particular patients are clinically unlikely to tolerate chemotherapy, for example, those who have pre-existing comorbid disease. These patients are candidates for palliative treatment $(8,9)$. Neither curative radical cystectomy nor chemotherapy was indicated in the current case due to distant metastases and a poor performance status, respectively.

Retroperitoneoscopic ureterocutaneostomy may relieve severe symptoms and bleeding from tumors, preventing urinary retention in the bladder. Furthermore, this procedure may avoid postrenal failure due to bilateral lymph node metastasis or locally advanced disease. It offers low morbidity with low-grade postoperative pain, and low probability of postoperative ileus compared with other urinary diversions using the intestinal tract, due to the use of retroperitoneal manipulation $(10,11)$. It appears to be a reasonable alternative to percutaneous nephrostomy for bilateral ureteral obstruction, provided that the clinical condition of the patient allows general anesthesia $(12,13)$. On the other hand, as it is a permanent and irreversible procedure, decisions regarding this option must be made following careful consideration of the individual's condition and risk assessment. Nakamura et al (14) performed retroperitoneoscopic ureterocutaneostomy in a patient with advanced bladder cancer who had a solitary kidney. Although the patient died of progressive disease 3 months after the operation, they did not present signs of postrenal failure nor did he complain of macrohematuria after the operation (14). In the present case, urinary diversion was performed only on the left side, as the right kidney was hydronephrotic, atrophic and nonfunctional, due to tumor obstruction. The left kidney functioned normally; therefore, the serum creatinine level did not change markedly perioperatively.

In conclusion, surgery under general anesthesia is typically avoided whenever possible in very elderly cases due to increasing operative risk. In particular, retroperitoneoscopic ureterocutaneostomy must be noted, as it is able to be performed in elderly patients due to it being a minimally invasive surgery without significant complications. Thus this surgery was able to be performed for the very elderly patient in the present case without postoperative delirium. Although its indication is limited to select cases, retroperitoneoscopic ureterocutaneostomy for very elderly patients of advanced bladder cancer with poor performance status may be an important procedure for alleviating symptoms and improving the quality of life.

\section{References}

1. Shariat SF, Sfakianos JP, Droller MJ, Karakiewicz PI, Meryn S and Bochner BH: The effect of age and gender on bladder cancer: a critical review of the literature. BJU Int 105: 300-308, 2010.

2. El-Tabey NA, Osman Y, Mosbah A, Mohsen T and Abol-Enein H: Bladder cancer with obstructive uremia: oncologic outcome after definitive surgical management. Urology 66: 531-535, 2005.

3. Ok JH, Meyers FJ and Evans CP: Medical and surgical palliative care of patients with urological malignancies. J Urol 174: 1177-1182, 2005.

4. Kearney GP, Docimo SG, Doyle CJ and Mahoney EM: Cutaneous ureterostomy in adults. Urology 40: 1-6, 1992.

5. MacGregor PS, Montie JE and Straffon RA: Cutaneous ureterostomy as palliative diversion in adults with malignancy. Urology 30: 31-34, 1987.

6. Ubrig B, Lazica M, Waldner M and Roth S: Extraperitoneal bilateral cutaneous ureterostomy with midline stoma for palliation of pelvic cancer. Urology 63: 973-975, 2004.

7. Gupta NP, Kolla SB, Seth A, Hemal AK, Dogra PN and Kumar R: Oncological and functional outcome of radical cystectomy in patients with bladder cancer and obstructive uropathy. J Urol 178: 1206-1211, discussion 1211, 2007.

8. Yi SK, Yoder M, Zaner K and Hirsch AE: Palliative radiation therapy of symptomatic recurrent bladder cancer. Pain Physician 10: 285-290, 2007.

9. Witjes JA, Compérat E, Cowan NC, De Santis M, Gakis G, Lebret T, Ribal MJ, Van der Heijden AG and Sherif A; European Association of Urology: EAU guidelines on muscle-invasive and metastatic bladder cancer: summary of the 2013 guidelines. Eur Urol 65: 778-792, 2014.

10. Yoshimura K, Ichioka K, Terada N, Matsuta Y, Okubo K and Arai Y: Retroperitoneoscopic tubeless cutaneous ureterostomy. BJU Int 89: 964-966, 2002.

11. Deliveliotis C, Papatsoris A, Chrisofos M, Dellis A, Liakouras C and Skolarikos A: Urinary diversion in high-risk elderly patients: modified cutaneous ureterostomy or ileal conduit? Urology 66: 299-304, 2005.

12. Puppo P, Perachino M, Ricciotti G and Bozzo W: Laparoscopic bilateral cutaneous ureterostomy for palliation of ureteral obstruction caused by advanced pelvic cancer. J Endourol 8: 425-428, 1994

13. Puppo P, Ricciotti G, Bozzo W, Pezzica C, Geddo D and Perachino M: Videoendoscopic cutaneous ureterostomy for palliative urinary diversion in advanced pelvic cancer. Eur Urol 28: 328-333, 1995.

14. Nakamura E, Terachi T, Kamoto T, Okuno H, Terai A, Kakehi Y and Ogawa O: Retroperitoneoscopic ureterocutaneostomy for obstructive uropathy with advanced bladder cancer: a case report. Int J Urol 9: 60-62, 2002. 